\title{
Effects of Exercise on Myocardial Force-Velocity Relations in Intact Unanesthetized Man: Relative Roles of Changes in Heart Rate, Sympathetic Activity, and Ventricular Dimensions *
}

\author{
Edmund H. Sonnenblick, $\dagger$ Eugene Braunwald, John F. Williams, JR., and \\ Gerald GLICK
}

(From the Cardiology Branch, National Heart Institute, Bethesda, Md.)

It is generally agreed that an increase in cardiac output is one of the primary circulatory adjustments that occurs during exercise. However, the nature of the alterations in the activity of the myocardium that permit this augmentation of output and the relative importance in the response to exercise of the various factors known to affect the performance of the heart and its contractile state has not been defined. It has been shown that the contractile state of cardiac muscle may be characterized by the force-velocity relation (1-3). The applicability of this concept to isolated human heart muscle has recently been demonstrated (4), and a technique has been described by which the force-velocity relation of the myocardium of intact human subjects can be evaluated (5), thus allowing determination of the effect of a variety of interventions on the contractile state of the heart. The purpose of the present investigation was to apply this technique in studying the effects of exercise on the force-velocity relations of the human heart, to define the relative roles of the changes in heart rate and of sympathetic activity that occur during exercise on the force-velocity relation, and to delineate the role of changes in ventricular end-diastolic size in the response of the heart to exercise.

\section{Methods}

Twelve studies were carried out in eleven patients ranging in age from 24 to 55 years (average $=44$ years). All had undergone cardiac operations consisting of the closure of an atrial septal defect in six patients, closure of a ventricular septal defect in two, aortic valve re-

\footnotetext{
* Submitted for publication April 22, 1965; accepted September 16, 1965.

$\dagger$ Address requests for reprints to Dr. Edmund H. Sonnenblick, Cardiology Branch, National Heart Institute, Bethesda, Md. 20014.
}

placement with a Starr-Edwards prosthesis in one, and mitral valvulotomy in two patients. At the time of cardiac operation small silver-tantalum markers were sutured to the right ventricle in seven patients, the left ventricle in two patients, and to the surface of both ventricles in two patients, as described in detail previously (6). Hemodynamic studies carried out 1 to 14 months postoperatively are summarized in Table $I$. The resting cardiac output was normal in all patients $(>2.50 \mathrm{~L}$ per minute per $\mathrm{m}^{2}$ ), as was the right atrial pressure, although the right ventricular end-diastolic pressure was slightly elevated in one patient (C.D.). The right ventricular systolic pressure was also slightly elevated in four patients (C.D., I.D., M.W., and S.C.).

The techniques for determining ventricular end-diastolic dimensions and the force-velocity relation have been described in detail elsewhere (5). At the time of postoperative catheterization, cineradiograms were exposed at 30 frames per second while intraventricular pressures were recorded simultaneously at a paper speed of $100 \mathrm{~mm}$ per second. A mechanical marker, activated by the $R$ wave of the electrocardiogram and recorded on the cineradiogram, permitted precise temporal correlation of ventricular dimensions and pressures. The distances between markers that had been placed in a line parallel to the frontal plane were measured on successive frames of the cineradiogram. All observations were made with the patient in the supine position.

The analysis of the force-velocity relation is predicated on the finding that throughout the active contraction the position of the force-velocity curve of the myocardium is determined by the instantaneous length of the muscle and by its contractile state (7). Thus, if the force-velocity relation is always examined at the same muscle length, this relation will define the contractile state of the myocardium. Accordingly, the force and velocity of a segment of the ventricle were always determined at the instant during the contraction when the segment passed through a specific length, termed the isolength point. It is recognized that the velocity of myocardial fiber shortening is not equal to the velocity of shortening of the contractile elements except when the series elastic component is not changing in length (8). However, the extension of the series elastic component takes place almost entirely during the isovolumetric phase of contraction 
TABLE I

Postoperative hemodynamic findings at rest*

\begin{tabular}{|c|c|c|c|c|c|c|c|c|c|c|c|}
\hline \multirow[b]{2}{*}{ Patient } & \multirow[b]{2}{*}{ Age, Sex } & \multirow[b]{2}{*}{ BSA } & \multirow[b]{2}{*}{ Diagnosis } & \multirow{2}{*}{$\begin{array}{l}\text { Time } \\
\text { after } \\
\text { oper- } \\
\text { ation }\end{array}$} & \multirow[b]{2}{*}{$\begin{array}{c}\text { Cardiac } \\
\text { index }\end{array}$} & \multicolumn{6}{|c|}{ Pressures } \\
\hline & & & & & & $\begin{array}{l}\text { RA } \\
(\mathrm{m})\end{array}$ & $\begin{array}{l}\text { RV } \\
\text { s/d }\end{array}$ & $\begin{array}{c}\text { PA } \\
\text { s/d (m) }\end{array}$ & $\begin{array}{l}\text { LA } \\
(\mathrm{m})\end{array}$ & $\begin{array}{l}\mathrm{LV} \\
\mathrm{s} / \mathrm{d}\end{array}$ & $\frac{\text { BA }}{\mathrm{s} / \mathrm{d}(\mathrm{m})}$ \\
\hline & & $m^{2}$ & & months & $\underset{u t e / m^{2}}{L / \min -}$ & \multicolumn{6}{|c|}{$m m \mathrm{Hg}$} \\
\hline J.S. & 33, M & 1.84 & P.O. VSD & 6 & 2.66 & 2 & $18 / 3$ & $16 / 5, \quad 11$ & & & $120 / 65,81$ \\
\hline G.N. & $55, M$ & 1.80 & P.O. AS & 6 & 2.53 & $\mathbf{0}$ & $19 / 0$ & $15 / 7, \quad 10$ & 3 & $124 / 6$ & $106 / 60,78$ \\
\hline S.J. & $28, M$ & 1.77 & P.O. VSD & 1 & 5.48 & 2 & $20 / 3$ & $19 / 5, \quad 10$ & & & $110 / 55,80$ \\
\hline C.D. & $43, F$ & 1.60 & P.O. MS & 12 & 2.74 & 4 & $32 / 7$ & $32 / 12,21$ & 14 & $160 / 9$ & $167 / 75,120$ \\
\hline K.G. & $27, \mathrm{M}$ & 1.89 & P.O. ASD & 13 & 3.76 & 0 & $10 /-1$ & $10 / 5,8$ & & & $132 / 75, \quad 96$ \\
\hline S.C. & $28, F$ & 1.52 & P.O. ASD & 13 & 4.01 & 1 & $44 / 1$ & $42 / 20,32$ & & & $115 / 60,85$ \\
\hline I.D. & $29, F$ & 1.56 & P.O. MS & 9 & 3.15 & 3 & $40 / 5$ & $39 / 19,28$ & 10 & $94 / 6$ & $98 / 60,77$ \\
\hline P.S. & $36, F$ & 1.66 & P.O. ASD & 4 & 4.64 & 2 & $29 / 3$ & $29 / 12,18$ & & & $140 / 65,82$ \\
\hline M.W. & $24, F$ & 1.70 & P.O. ASD & 14 & 2.91 & 5 & $34 / 5$ & $23 / 8, \quad 15$ & & & $128 / 70,95$ \\
\hline R.W. & 35, M & 1.93 & P.O. ASD & 8 & 3.64 & 3 & $20 / 4$ & $20 / 8, \quad 12$ & & & $133 / 64,81$ \\
\hline A.H. & $39, \mathrm{~F}$ & 1.50 & P.O. ASD & 7 & 4.09 & $\mathbf{0}$ & $24 / 1$ & $23 / 5, \quad 10$ & & & $106 / 54,72$ \\
\hline
\end{tabular}

*Abbreviations: P.O. = postoperative, VSD = ventricular septal defect, ASD = atrial septal defect, AS = aortic stenosis with Starr-Edwards prosthetic valve replacement, $\mathrm{MS}=$ mitral stenosis, $\mathrm{RA}=$ right atrium, $\mathrm{RV}=$ right ventricle, PA $=$ pulmonary artery, LA $=$ left atrium,
$\mathrm{LV}=$ left ventricle, $\mathrm{BA}=$ brachial artery, $\mathrm{m}=$ mean, and $\mathrm{s} / \mathrm{d}=$ systolic/diastolic.

and is virtually complete at the point during the ejection phase at which the measurements were made. Thus, it is unlikely that alterations of the rate of elongation of the series elastic component could have altered the relationship between the velocity of shortening of the segment of myocardium and of the contractile elements.

The instantaneous distances between the markers on the ventricular surface were plotted below the intraventricular pressures of the same cardiac cycles, as shown in Figure 1. The contour of the dimension curve and temporal relationships between intraventricular pressure and dimensions have been shown to be similar for both ventricles $(6,9)$. The isolength point was selected as a length of the segment of myocardium that was common to all cardiac cycles and that occurred during the first two-thirds of ventricular ejection. The rate at which the markers approached each other was determined when the segment of myocardium passed through this isolength point (Figure 1, points $a$ and $a^{\prime}$ ), and the instantaneous velocity of shortening was determined by measuring the tangent to the curve relating ventricular length to time at this point (Figure 1 , points $b$ and $b^{\prime}$ ). Whereas intramyocardial wall tension could not be measured directly by this technique, it is known from the LaPlace relation (10) that, at any one ventricular volume, wall tension is a direct function of intraventricular pressure. Therefore, to use intraventricular pressure as a direct reflection of myocardial wall tension, ventricular pressure was measured relative to the same isolength point (Figure 1, points $c$ and $c^{\prime}$ ).

The distances between the markers and the velocity with which they approached each other were initially expressed in centimeters and centimeters per second, respectively. Distances between markers could be reproducibly measured within a $1 \%$ error (6). However, these measurements represent relative distances and velocities, since the absolute values depend on the magnification occurring when the markers are projected on a screen. Since the degree of magnification was constant during any given set of analyses, it was possible to express velocity of shortening in units of "muscle lengths per second" by dividing the velocity in centimeters per second by the isolength measurement in centimeters. Six to fifteen complete cardiac cycles were analyzed during each condition, and the average velocities of shortening and pressures for these cycles are presented in Table II. As described previously, an increase in the velocity of shortening at any level of intraventricular pressure, i.e., an upward displacement of the force-velocity relation, denotes an augmentation of the contractile state of the myocardium (5).

The effects of light exercise performed on a bicycle ergometer while the patient was in the supine position were studied in all 11 patients. The contribution of heart rate to the exercise response was studied in six patients (experiments 1 to 6 ). The heart rate attained during the normal bout of exercise was recorded, and after recovery, with the patient at rest, the right atrium was stimulated with an electrode catheter in a manner described elsewhere (11) at the rate previously attained during exercise, and measurements of force-velocity relations were repeated. The patient was then exercised again while electrical stimulation of the atrium was continued, and the force-velocity relation was redetermined.

The contribution of the sympathetic nervous system to the response of the heart to exercise was studied in six patients (No. 7 to 12). After recovery from an initial bout of exercise, each patient received propranolol ${ }^{1}(0.17$ mg per $\mathrm{kg}$ ), a beta-adrenergic blocking agent (12). Five minutes later, an identical bout of exercise was repeated. In two patients (No. 10 and 11 ), total body $\mathrm{O}_{2}$ consumption was measured to ensure that the levels of exercise were similar before and after sympathetic blockade. In three patients (No. 7, 8, and 10), $2 \mathrm{mg}$ atropine was given intravenously to inhibit the vagal response during

$1 l$-Isopropylamino-3-( $l$-naphthyloxy) -2-propanol hydrochloride. 
exercise, and both control and exercise measurements were repeated. In one patient (No. 12) the effects of beta blockade on the response to exercise were determined at a constant heart rate, as described above.

In three patients (No. 8-10), the rate of right ventricular pressure development was measured with a Allard-Laurens micromanometer catheter system and linear resistance-capacitance network differentiating circuit, as described previously (13). In every experiment, each determination of the force-velocity relation was accom- panied by a measurement of cardiac output by the indicator-dilution method as detailed elsewhere (14).

\section{Results}

Effects of spontaneous exercise. During the initial bout of exercise the cardiac response was allowed to occur spontaneously, without control of heart rate or of autonomic nervous activity. Car-

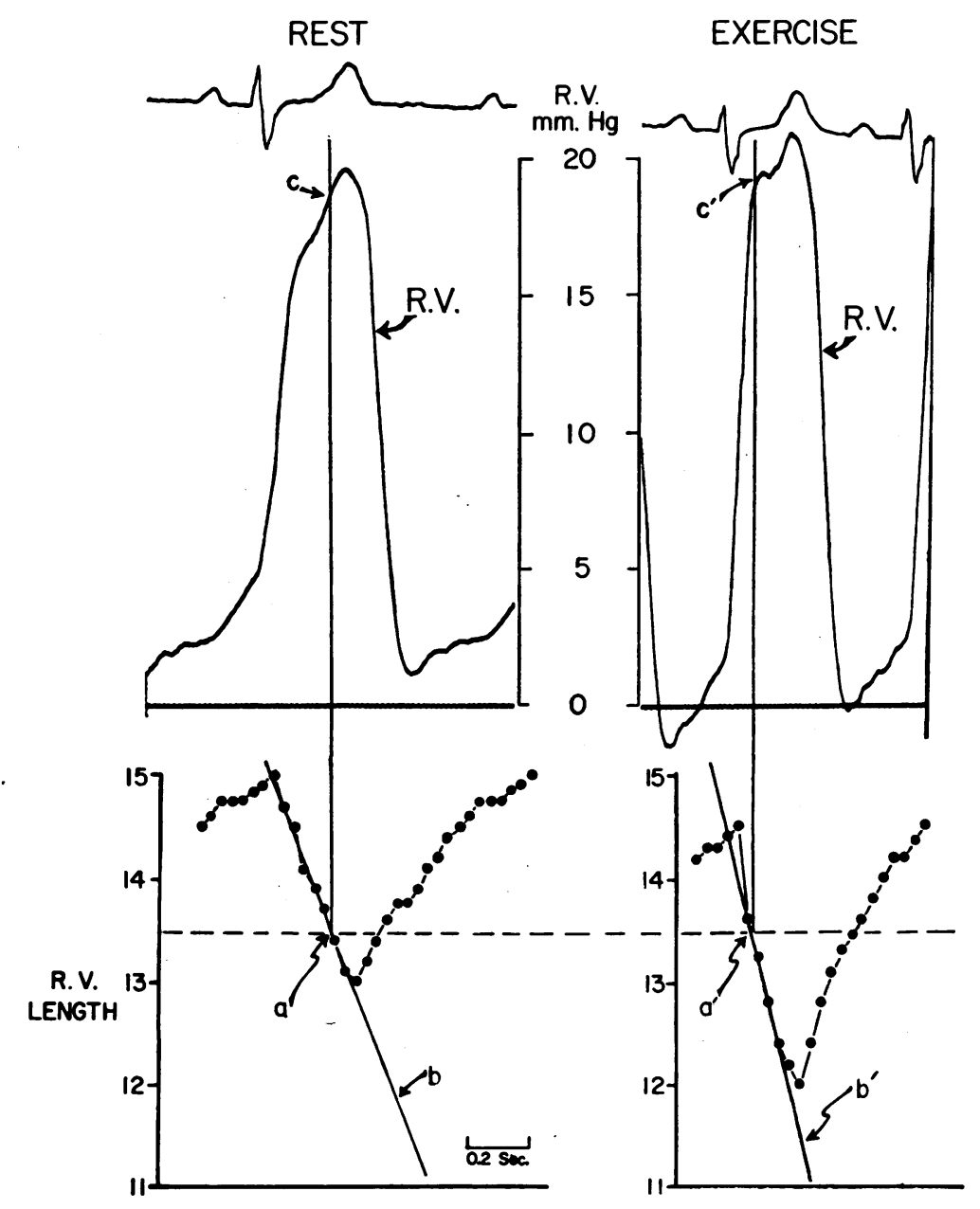

Fig. 1. MEthod FOR DETERMining the instantaneous Myocardial FORCE-VELOCITY RELATION ON A BEAT-TO-BEAT BASIS. From above downwards are shown the electrocardiogram, the right ventricular (RV) pressure pulse, and the curve relating right ventricular dimensions, determined at 1 - to 30 second intervals, to time. On the left are the data obtained during the control period and on the right the observations made during exercise. Points $a$ and $a^{\prime}$ represent the isolength points at which both instantaneous velocity of shortening and intraventricular pressure are determined. Line $b$ and $b^{\prime}$ are the tangents to the length curves at points $a$ and $a^{\prime}$ and represent the velocity of shortening at these points. The steeper slope of $b^{\prime}$ as compared to $b$ signifies an augmentation of velocity. Points $c$ and $c^{\prime}$ represent the temporally related points on the ventricular pressure curve. 


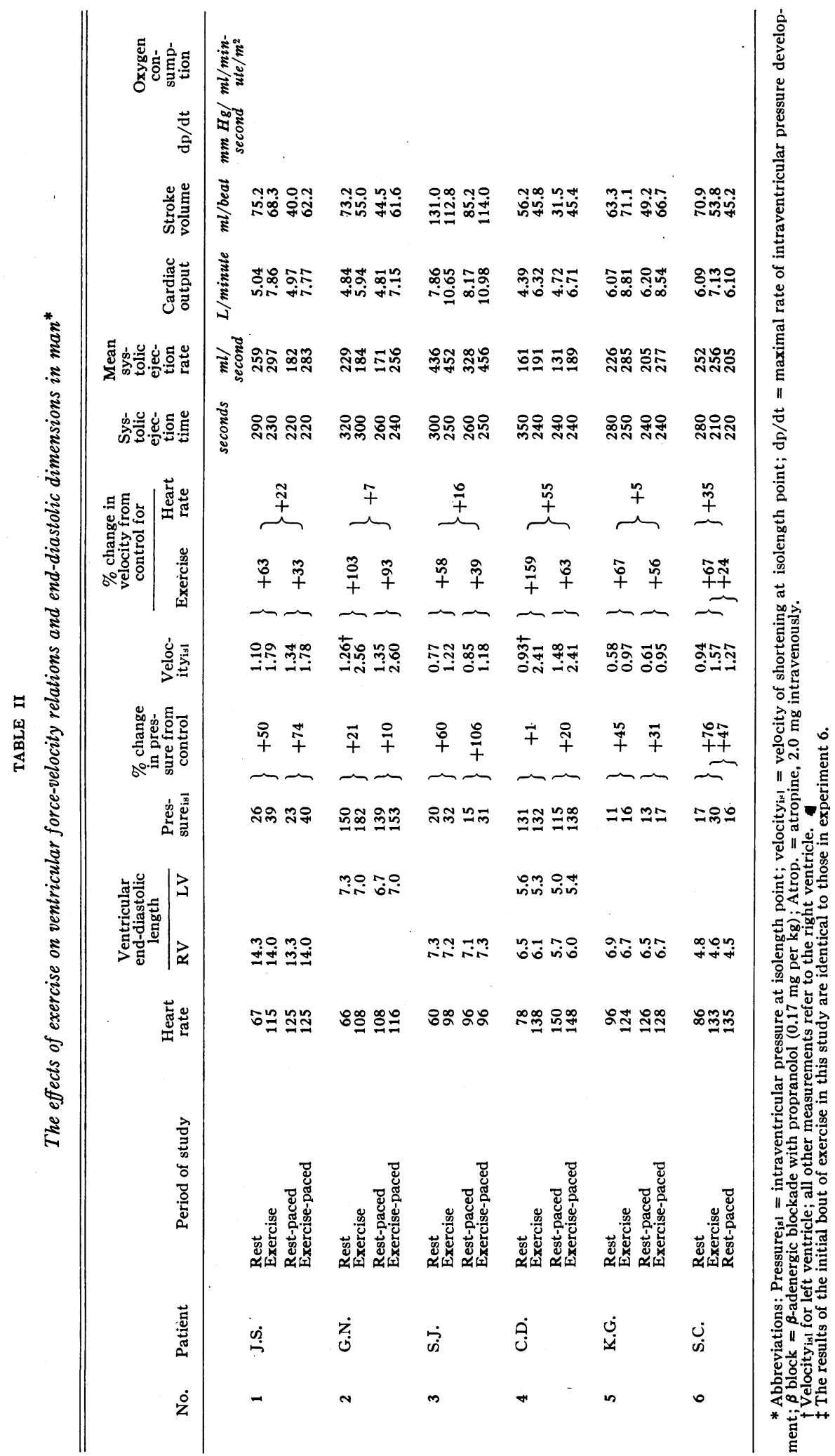


EXERCISE AND MYOCARDIAL FORCE-VELOCITY RELATIONS

2055




diac output rose from an average value at rest of $6.03 \mathrm{~L}$ per minute to $8.17 \mathrm{~L}$ per minute, and heart rate increased from an average level of 74 per minute to 111 per minute, whereas stroke volume declined slightly from an average of $83.7 \mathrm{ml}$ per minute to $76.5 \mathrm{ml}$ per minute. The contractile state of the heart was augmented, as attested to by the shift of the force-velocity relation upward and to the right. Ventricular pressure, measured at the time of the isolength point (pressure ${ }_{\text {isl }}$ ) increased in 10 of the 11 patients ; in the eight studies on the right ventricle, it rose from an average value of 21.8 to $28.8 \mathrm{~mm} \mathrm{Hg}(+32 \%$ ), whereas in the three studies on the left ventricle it rose from an average value of 124 to $136 \mathrm{~mm} \mathrm{Hg}$
$(+10 \%)$. In all 11 studies the instantaneous velocity of shortening, also measured at the isolength point $\left(\right.$ velocity $_{\text {isl }}$ ) increased from an average of 0.84 muscle lengths per second to an average of 1.51 muscle lengths per second, representing an average increase of $80 \%$. In every patient ventricular end-diastolic length decreased with spontaneous exercise diminishing by an average of $3.1 \%$ in the left ventricle in four patients and by $4.3 \%$ in the right ventricle in nine patients. Examples of cardiac dimensions at rest and during spontaneous exercise are illustrated in Figure 2, panels $A$ and $B$, and Figure 4, panels $A$ and $B$, whereas the shift in the force-velocity relation up-



Fig. 2. THE EFFECTS OF CONTROLling heART RATE ON THE END-DIASTOLIC DIMENSIONS OF THE HEART AND THE COURSE OF VENTRICULAR CONTRACTION DURING EXERCISE. The distances between markers on the right ventricle as a function of time for single contractions are shown for the study in patient J.S. Panel A represents the resting state; panel $\mathrm{B}$ is during spontaneous exercise. The broad dashed line at the top labeled RVEDL represents the end-diastolic dimensions at rest. $\mathrm{RVESL}=$ right ventricular end-systolic length. Panel $\mathrm{C}$ is during electrical stimulation of the right atrium with the subject at rest, and panel $\mathrm{D}$ is during exercise performed at the same heart rate. The top broken line in panels $C$ and $D$ corresponds to the RVEDL in panel A. H.R. = heart rate, C.O. = cardiac output, and S.V. = stroke volume. 
wards and to the right during these two bouts of exercise is shown in Figures 3 and 5.

Effects of exercise at a constant heart rate. After study of the effects of spontaneous exercise with the patient at rest, the heart rate was increased to that level attained during the previous bout of exercise. This augmentation of heart rate alone resulted in no significant change in the cardiac output (average $=+1.6 \%$ ), whereas a considerable decrease in stroke volume was noted (average $=-37 \%$ ). Increasing heart rate in the resting state resulted in a decrease in the enddiastolic ventricular dimensions to levels that were smaller than those observed during spontaneous exercise at a comparable heart rate (Figure 2, panels $\mathrm{B}$ and $\mathrm{C}$ ). Tachycardia with the patient at rest increased velocity isl $_{\text {by }}$ an average of $23 \%$, whereas the changes in pressure isl $_{\text {were small and }}$ inconstant. Thus, the extent of the shift in the force-velocity relation resulting from an increase in heart rate alone was considerably less than that observed when similar heart rates were achieved in the same patients during exercise where the velocity $_{\text {isl }}$ increased by an average of $89 \%$.

When the exercise was repeated at a constant heart rate, cardiac output and stroke volume rose to the levels attained during the first bout of exercise. However, rather than a decrease, an increase in ventricular end-diastolic dimensions now occurred, averaging 9\% (Figure 2, panels $\mathrm{C}$ and D). The contractile state of the myocardium was again augmented during this bout of exercise; velocity $_{\text {isl }}$ rose by an average of $57 \%$ and pressure $_{\text {ist }}$ increased an average of $47 \%$, even though heart rate was held constant (Figure 3 ).

Effects of exercise during beta-adrenergic blockade. After the study of the effects of spontaneous exercise, propranolol was administered to six patients (Table II, experiments 7 to 12). Slight decreases in resting heart rate (average $=-12 \%$ of control) and cardiac output (average $=-19 \%$ of control) were observed, whereas little change in total $\mathrm{O}_{2}$ consumption occurred in the two patients in whom it was measured. No consistent changes in stroke volume or ventricular end-diastolic dimensions were observed at rest (Figure 4, panels $A$ and $C$ ). Beta-adrenergic blockade did not appear to affect the myocardial contractile state under resting conditions significantly; velocity $_{\text {isl }}$ changed by an average of only $-7 \%$ while pres-

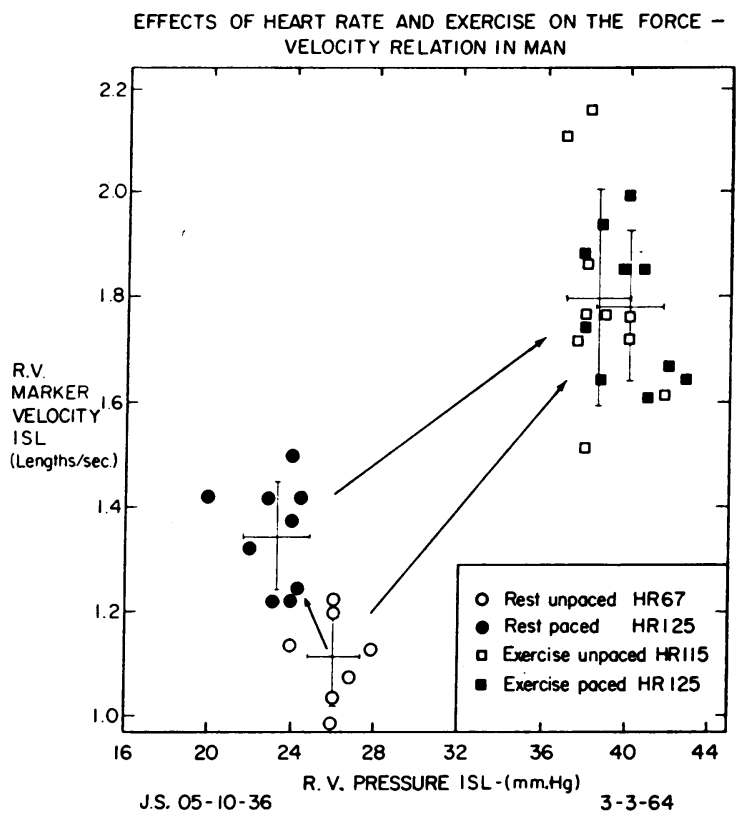

Fig. 3. The effects of heart RATE AND EXerCise oN THE FORCE-VELOCITY RELATIONS OF THE VENTRICLE FROM the SAME EXPERIMENT AS illustrated in Figure 2. On the ordinate is the velocity of shortening of the segment of myocardium between the markers at isolength, and on the abscissa is the corresponding right ventricular pressure. Each symbol represents a single ventricular contraction. The crossbars represent $1 \mathrm{SD}$. ISL= isolength.

sure $_{\text {isl }}$ rose by an average of $3 \%$. This conclusion was further substantiated by the finding that the rate of ventricular pressure development $(\mathrm{dp} / \mathrm{dt})$ was little altered in the three experiments in which it was measured (Table II, no. 8 to 10).

When exercise was repeated after beta-adrenergic blockade, the augmentation of cardiac output was comparable to that observed before propranolol in two studies (no. 7 and 9). The increase was substantially less in three studies (no. 10 to 12), and in one study (no. 8) cardiac output failed to rise after beta blockade. Despite beta-adrenergic blockade, heart rate rose with exercise, although the increments and absolute levels achieved were lower than during the control bout of exercise. In contrast to exercise in the unblocked state, ventricular end-diastolic dimensions now failed to decline. Little augmentation of the contractile state was observed during exercise after beta-adrenergic blockade; velocity of shortening ${ }_{\text {isl }}$ increased by an average of only $10 \%$, as compared with an average increase of $69 \%$ in the same pa- 


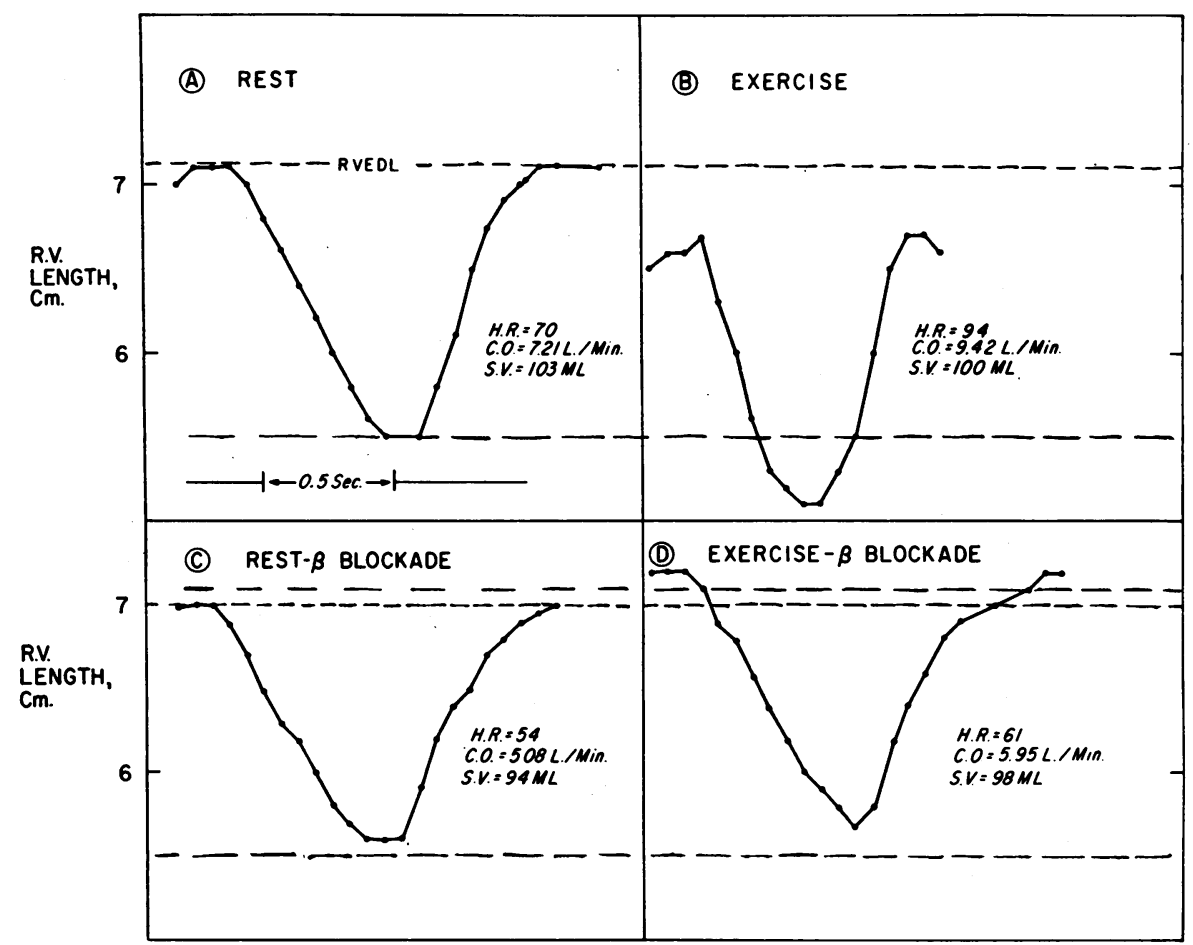

Fig. 4. THE EFFECTS OF BETA-ADRENERGIC BLOCKADE ON THE RESPONSE OF THE HEART TO EXERCISE. The distance between right ventricular markers during individual contractions are shown as a function of time for the study in patient A.H. $A=$ control state, $\mathrm{B}=$ spontaneous exercise, $\mathrm{C}=$ rest after propranolol, and $\mathrm{D}=$ exercise after propranolol.

EFFECTS OF $\beta$-BLOCKADE ON THE RESPONSE OF FORCE-VELOCITY RELATION TO EXERCISE

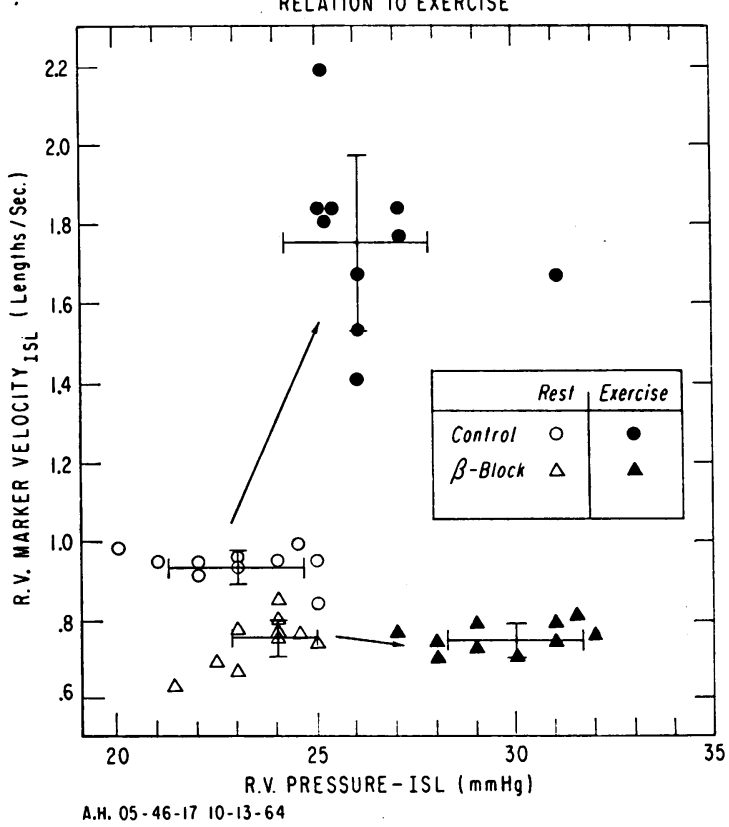

Fig. 5. EFFECTS OF BETA-ADRENERGIC BLOCKADE ON THE RESPONSE OF THE FORCE-VELOCITY RELATION TO EXERCISE FOR THE STUdy illustrated IN Figure 4. tients during the initial control exercise period, whereas the changes in pressure isl $_{\text {were comparable }}$ in the blocked and unblocked states.

In four patients an attempt was made to study the effects of exercise after beta blockade without accompanying changes in heart rate. This was entirely successful in experiment 12 (Figure 6), in which electrical stimulation was employed, whereas in experiments 7,8 , and 10 , in which atropine was given, the heart rate still increased during exercise, although to a smaller extent than during beta blockade alone. In these experiments ventricular end-diastolic dimensions again did not decline during exercise, but actually tended to increase (Table II). More striking, however, was the finding that the increase in velocity ${ }_{\text {isl }}$ was blocked; this variable changed by an average of only $+3 \%$ in the four studies.

\section{Discussion}

Previous investigations on the circulatory effects of exercise have placed primary emphasis on the over-all response of the heart as a pump, whereas the major objective of the present study 
was to examine the effects of this stimulus on the characteristics of the heart viewed as a muscle. It is now widely appreciated that the circulatory changes that occur during exercise in man are dependent upon the intensity of the stress as well as on the position of the body during the exercise. In this investigation the effects of relatively mild muscular effort carried out in the supine position were studied. It has been shown previously that under these circumstances the increase in cardiac output is mediated primarily by an increase in heart rate with a relatively constant stroke volume $(15,16)$, whereas ventricular end-diastolic dimensions decline $(9,17)$. The findings of the present study are in accord with these views.

In this investigation it was observed that the well-known responses of the pumping action of the heart are accompanied by striking alterations in the contractile state of the myocardium. This was shown by a marked shift of the instantaneous force-velocity relations of the myocardium. Average velocity $y_{\text {isl }}$ increased $80 \%$, although this mild level of exercise induced only modest elevations of cardiac output (average $=36 \%$ ) and no significant increases in stroke volume. Thus, even though the performance of the ventricle as a pump was not altered strikingly, the shift of the forcevelocity relation indicates that the exercise state induced an elevation of the velocity of fiber shortening at any given intraventricular pressure and dimension.

It is appreciated that determination of the rate of shortening of a segment of the ventricle, as carried out in this and the previous study (5), provides a measurement of the velocity of shortening of the myocardial fibers but not of the contractile elements. From a consideration of studies carried out both on skeletal muscle $(18,19)$ and on the isolated papillary muscle $(2,3)$, it is clear that the contractile element velocity is identical to the myocardial fiber velocity only when myocardial force is constant. Furthermore, the physical characteristics of the series elastic component in heart muscle have been defined (20), allowing calculation of the differences between the velocities of shortening of myocardial fibers and of the contractile elements. Levine and Britman (8) have compared the shortening velocities throughout systole in the canine left ventricle and have demonstrated that the major discrepancy between them occurs during isometric contraction and dur-

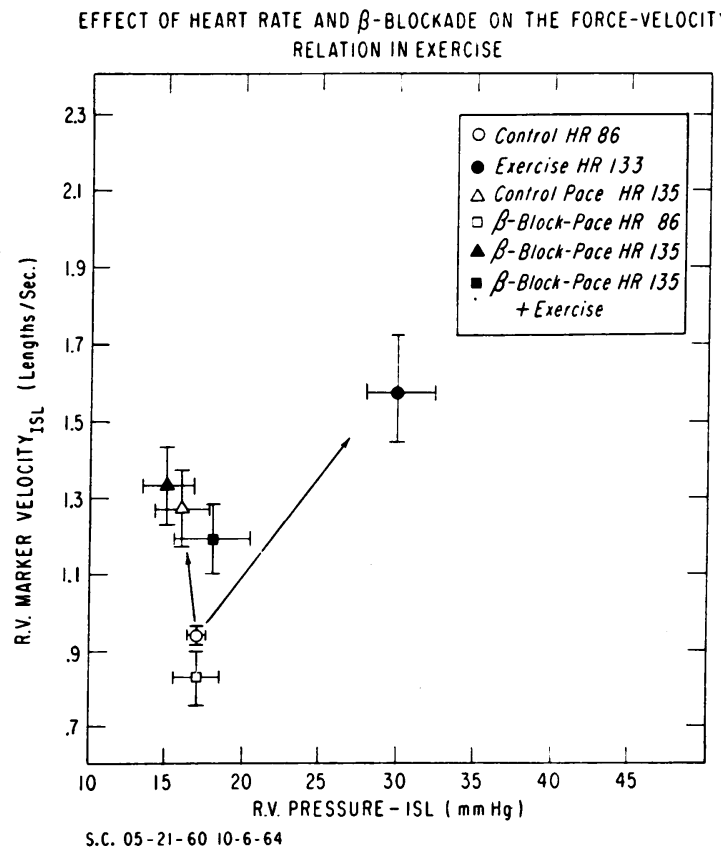

Fig. 6. THE EFFECTS OF HEART RATE AND BETA-ADRENERGIC BLOCKADE ON THE FORCE-VELOCITY RELATION DURING THE EXERCISE RESPONSE. The symbols denote the average values for pressure and velocity; the crossbars represent $1 \mathrm{SD}$.

ing the first $30 \mathrm{msec}$ of ejection, a time when myocardial force is changing rapidly. Thereafter, since force changes much less rapidly, the velocities of shortening of the myocardial fibers and contractile elements are quite similar although not identical. Similar conclusions were reached by Fry, Griggs, and Greenfield (21). In a more recent study from this laboratory on the canine heart (22), specific comparisons were made between the velocities of shortening of myocardial fibers and contractile elements at constant ventricular volumes. A close correlation was found during the midportion of ejection at a variety of afterloads; in 14 experiments carried out on seven dogs the contractile element velocity exceeded the velocity of fiber shortening by an average of $9.0 \pm$ $1.5 \%$ (SE). Furthermore, the close relationship between these variables was not altered during the profound inotropic stimulation provided by infusion of norepinephrine (22). Thus, although techniques for the direct measurement of contractile element velocity in the intact human heart are not available, the theoretical considerations and experimental findings presented above indicate that a useful and reasonably reliable estimate of this variable can be obtained by determining 
myocardial fiber velocity. Since the measurements in the present study were made at a time during the midportion of ejection when the rates of change in the length of the series elastic components of the muscle are minimal $(8,21)$, it is likely that the observed increase in the velocity of fiber shortening during exercise reflects an augmentation of contractile element velocity, which implies that the rates of the chemical reactions responsible for the generation of force and shortening have also been increased (23).

In an attempt to elucidate the mechanism responsible for the marked shift in the force-velocity relation that occurred during exercise, consideration was given to the possible roles played by alterations in heart rate and sympathetic activity, since previous studies had shown that tachycardia as well as infusion of sympathomimetic amines shifted the force-velocity relation in a similar manner (5). It was observed in this investigation that tachycardia with the subject at rest shifted the force-velocity relation to a relatively small extent in comparison to the shifts produced by exercise. However, since pressure isl $_{1}$ tended to decrease with increments in heart rate alone, some of the increments in fiber shortening rate that were observed might reflect the reduction in afterload rather than a shift in the force-velocity relations of the myocardium. Furthermore, when exercise was performed at a constant heart rate it was observed that the major shift of the force-velocity relation occurred then (Figure 3 ). Thus, although the increase in heart rate observed during spontaneous exercise helps to explain the relative constancy of stroke volume, it does not play an important role in the increase of the contractile state of the myocardium that occurs.

The possibility that the exercise-induced increase in the velocity of contraction resulted from sympathetic stimulation of the myocardium has been investigated with the aid of the beta-adrenergic blocking drug propranolol. This agent has been shown to block adrenergic receptors in the myocardium in doses that do not exert a direct depressant effect on the heart (24), and, in the dose used in the present investigation, to produce a moderate reduction of cardiac output both during maximal and submaximal levels of exercise (25). It was observed in this investigation that beta blockade prevented the increase in the exer- cise-induced shift in the force-velocity relation almost completely. The small residual increases in the contractile state that occurred after propranolol could be attributed to the elevation of heart rate that still occurred, since when heart rate was also controlled even these small shifts were abolished (Figure 6).

The finding that the administration of propranolol does not alter the contractile state of the myocardium at rest, apart from the changes resulting from the slowing of heart rate, lends support to the view that there is relatively little basal sympathetic stimulation of the heart in supine, resting individuals (26). On the other hand, the marked effect of the drug on the force-velocity relation during exercise indicates that the sympathetic system profoundly affects the contractile state of the myocardium at this time. Despite the loss of the normal exercise-induced shift in the contractile state after beta blockade, exercise could still be performed with some rise in the cardiac output. Thus, it is clear that mechanisms are still available to increase the performance of the ventricle as a pump even when the improvement in the contractile state of the myocardium is blocked.

In studies on the circulatory adaptations to exercise there has long been an interest in the effects of this stimulus on ventricular volume and dimensions. The finding that the end-diastolic size of the heart usually does not increase and, in fact, most commonly declines $(9,27-31)$, has been used to support the view that the Frank-Starling mechanism does not play a role in the cardiac response to exercise (32). It is clear from the present study that induced tachycardia in the absence of exercise reduces ventricular dimensions to a greater extent than do similar changes in heart rate during exercise. In other words, at any given heart rate ventricular end-diastolic dimensions, end-systolic dimensions, and stroke volume are all larger during exercise than at rest. Thus, the Frank-Starling mechanism does in fact participate in the adaptation of the heart to exercise, even in the presence of an actively functioning sympathetic nervous system. However, the increase in enddiastolic volume is not seen because of the opposing effects produced by the tachycardia per se. In addition to tachycardia the increased sympathetic activity also contributes to the decrease in end-diastolic dimensions during exercise, since 
after propranolol exercise no longer reduced enddiastolic size (Figure 4). This latter conclusion is consonant with studies on denervated dogs (31, 33 ) in which exercise resulted in increases in ventricular end-diastolic volume as well as stroke volume.

Previous studies have indicated that the systolic ejection rate reflects the velocity of myocardial contraction $(34,35)$. However, from the present study it is apparent that these measurements do not necessarily reflect a change in the rate of myocardial fiber shortening. In accord with the findings of other investigators $(11,34)$, increments in heart rate alone have been found to abbreviate the sytolic ejection time significantly (Table II). However, this abbreviation of the systolic ejection time was accompanied by only minor changes in the velocity of myocardial fiber shortening (Table II). Furthermore, when exercise was performed in the absence of a change of heart rate, only small or inconstant changes in the systolic ejection time were found, although large increments in the velocity of myocardial fiber shortening occurred (Table II). Similarly, the mean systolic ejection rate, i.e., the stroke volume divided by the duration of ejection, did not reflect the velocity of myocardial fiber shortening in all instances, since significant increments in this variable occurred in the presence of beta-adrenergic blockade that prevented the augmentation of the contractile state of the ventricle resulting from sympathetic stimulation.

In conclusion, the present investigation carried out on intact, conscious subjects has allowed an analysis of the interplay that occurs between three fundamental adaptive mechanisms available to the heart during exercise: 1) augmentation of the contractile state of the myocardium, 2) increase in heart rate, and 3) augmentation of the force of contraction ensuing from an increase of end-diastolic dimensions (Frank-Starling mechanism). During relatively mild exertion in the supine position a marked improvement of the contractile state of the heart occurs, as evidenced by a shift in the myocardial force-velocity relation. This shift allows the ventricle to eject blood more rapidly at any given force. The augmentation of the contractile state is mediated primarily by an increase in sympathetic nervous activity, and only to a minor extent by the increase of heart rate per se.
On the other hand, the decrease in ventricular enddiastolic dimensions observed during spontaneous exercise may be explained primarily by the accompanying tachycardia. Since exercise carried out at a constant heart rate actually increases stroke volume and end-diastolic dimensions, the Frank-Starling mechanism is operative but overshadowed by the increase in heart rate.

\section{Summary}

The responses of the heart to mild exercise were studied in 11 patients utilizing a cineradiographic technique, which allows an evaluation of myocardial force-fiber shortening rate relations as well as cardiac dimensions. The technique consisted of exposing cineradiograms at 30 frames per second and measuring the velocity of movement of roentgenopaque markers that had been sutured to the external surface of the ventricles while simultaneously recording intraventricular pressure. A beat-to-beat analysis of the force-velocity relation was then accomplished by measuring the velocity and the pressure at a constant length point in each contraction. It was observed that exercise resulted in an augmentation of the contractile state of the myocardium as evidenced by an increase in the velocity of contraction at any given force, and that this shift of the force-velocity relation could be blocked almost completely with the beta-adrenergic receptor blocking agent propranolol. The contribution of heart rate changes to this shift in the force-velocity relation was minor. Tachycardia induced without exercise reduced ventricular end-diastolic dimensions to a greater extent than exercise, thus providing evidence for the participation of the Frank-Starling mechanism during exercise. It is concluded that the response of the heart to exercise results from a synthesis of three fundamental adaptive mechanisms: 1) shift of the myocardial force-velocity relation, 2) tachycardia, and 3) the Frank-Starling mechanism.

\section{References}

1. Abbott, B. C., and W. F. H. M. Mommaerts. A study of inotropic mechanisms in the papillary muscle preparation. J. gen. Physiol. 1959, 42, 533.

2. Sonnenblick, E. H. Force-velocity relations in mammalian heart muscle. Amer. J. Physiol. 1962, 202, 931.

3. Sonnenblick, E. H. Implications of muscle mechanics in the heart. Fed. Proc. 1962, 21, 975. 
4. Sonnenblick, E. H., E. Braunwald, and A. G. Morrow. The contractile properties of human heart muscle: studies on myocardial mechanics of surgically excised papillary muscles. J. clin. Invest. 1965, 44, 966.

5. Glick, G., E. H. Sonnenblick, and E. Braunwald. Myocardial force-velocity relations studied in intact unanesthetized man. J. clin. Invest. 1965, 44, 978.

6. Harrison, D. C., A. Goldblatt, and E. Braunwald. Studies on cardiac dimensions in intact, unanesthetized man. I. A description of techniques and their validation. Circulat. Res. 1963, 13, 448.

7. Sonnenblick, E. H. Instantaneous force-velocitylength determinants in the contraction of heart muscle. Circulat. Res. 1965, 16, 441.

8. Levine, H. J., and N. A. Britman. Force-velocity relations in the intact dog heart. J. clin. Invest. 1964, 43, 1383.

9. Braunwald, E., A. Goldblatt, D. C. Harrison, and D. T. Mason. Studies on cardiac dimensions in intact, unanesthetized man. III. Effects of muscular exercise. Circulat. Res. 1963, 13, 460.

10. Burton, A. C. The importance of the shape and size of the heart. Amer. Heart J. 1957, 54, 801.

11. Ross, J., Jr., J. W. Linhart, and E. Braunwald. Effects of altering heart rate by electrical stimulation of the right atrium in man: studies at rest, during muscular exercise, and isoproterenol infusion. Amer. J. Physiol. In press.

12. Black, J. W., A. F. Crowther, R. G. Shanks, L. H. Smith, and A. C. Dornhorst. A new adrenergic beta-receptor blocking antagonist. Lancet 1964, 2, 1080.

13. Gleason, W. L., and E. Braunwald. Studies on the first derivative of the ventricular pressure pulse in man. J. clin. Invest. 1962, 41, 80 .

14. Braunwald, E., and E. R. Kelly. The effects of exercise on central blood volume in man. J. clin. Invest. 1960, 39, 413.

15. Rushmer, R. F. Constancy of stroke volume in ventricular responses to exertion. Amer. J. Physiol. 1959, 196, 745.

16. Wang, Y., R. J. Marshall, and J. T. Shepherd. The effect of changes in posture and of graded exercise on stroke volume in man. J. clin. Invest. 1960, 39, 1051.

17. Williamson, C. S. The effects of exercise on the normal and pathological hearts; based upon the study of one hundred cases. Amer. J. med. Sci. $1915,149,492$.

18. Hill, A. V. The heat of shortening and the dynamic constants of muscle. Proc. roy. Soc. B 1938, 126, 136.

19. Jewell, B. R., and D. R. Wilkie. An analysis of the mechanical components in frog's striated muscle. J. Physiol. (Lond.) 1958, 143, 515.

20. Sonnenblick, E. H. Series elastic and contractile elements in heart muscle: changes in muscle length. Amer. J. Physiol. 1964, 207, 1330.
21. Fry, D. L., D. M. Griggs, Jr., and J. C. Greenfield, Jr. Myocardial mechanics: tension-velocity-length relationships of heart muscle. Circulat. Res. 1964, 14, 73.

22. Ross, J., Jr., J. W. Covell, E. H. Sonnenblick, and E. Braunwald. Contractile state of the heart characterized by force-velocity relations in variably afterloaded and isovolumic beats. Circulat. Res. In press.

23. Podolsky, R. J. Mechanochemical basis of muscular contraction. Fed. Proc. 1962, 21, 964.

24. McInery, T. K., D. P. Gilmour, and J. R. Blinks. Comparison of effects of propranolol and other cardiac adrenergic blocking agents on inotropic and chronotropic actions of catecholamines (abstract). Fed. Proc. 1965, 24, 712.

25. Epstein, S. E., B. F. Robinson, R. L. Kahler, and E. Braunwald. Effects of beta-adrenergic blockade on the cardiac response to maximal and submaximal exercise in man. J. clin. Invest. 1965, 44, 1745.

26. Glick, G., and E. Braunwald. Relative roles of the sympathetic and parasympathetic nervous systems in the reflex control of heart rate. Circulat. Res. $1965,16,363$.

27. Liljestrand, G., E. Lysholm, and G. Nylin. The immediate effects of muscular work on the stroke and heart volume in man. Skand. Arch. Physiol. 1938, 80, 265.

28. Kjellberg, S. R., U. Rudhe, and T. Sjöstrand. The amount of hemoglobin (blood volume) in the relation to the pulse rate and heart volume during work. Acta physiol. scand. 1950, 19, 152.

29. Ruosunoja, R., E. Linko, J. Lind, and A. Sollberger. Heart volume changes at rest and during exercise. Acta med. scand. 1958, 162, 263.

30. Chapman, C. B., O. Baker, and J. H. Mitchell. Left ventricular function at rest and during exercise. J. clin. Invest. 1959, 38, 1202.

31. Bruce, T. A., C. B. Chapman, O. Baker, and J. N. Fisher. The role of autonomic and myocardial factors in cardiac control. J. clin. Invest. 1963, 42, 721.

32. Rushmer, R. F., O. Smith, and D. Franklin. Mechanisms of cardiac control in exercise. Circulat. Res. 1959, 7, 602.

33. Donald, D. E., and J. T. Shepherd. Initial cardiovascular adjustment to exercise in dogs with chronic cardiac denervation. Amer. J. Physiol. 1964, 207, 1325.

34. Weissler, A. M., P. G. Peeler, and W. H. Roehil. Relationships between left ventricular ejection time, stroke volume, and heart rate in normal individuals and patients with cardiovascular disease. Amer. Heart J. 1961, 62, 367.

35. Levine, H. J., W. A. Neill, R. J. Wagman, H. Krasnow, and R. Gorlin. The effect of exercise on mean left ventricular ejection rate in man. J. clin. Invest. 1962, 41, 1050. 